\title{
Health Behavior Change Interventions for Teenage and Young Adult Cancer Survivors: A Systematic Review
}

\author{
Gemma Pugh, Helen L. Gravestock, MA, ${ }^{2}$ Rachael E. Hough, MD, \\ Wendy M. King, MSc, Jane Wardle, $\mathrm{PhD}$, and Abigail Fisher, $\mathrm{PhD}^{1}$
}

Purpose: It is important that teenage and young adult (TYA) cancer survivors adopt a healthy lifestyle, since health vulnerabilities associated with their diagnosis and treatment may be exacerbated by poor health behaviors. This review aims to synthesize the current literature on health behavior change interventions created specifically for TYA-aged cancer survivors.

Method: MEDLINE, EMBASE, PsycINFO, and CINAHL databases were searched for studies investigating interventions targeting one or more health behaviors, including: physical activity, diet, smoking cessation, and alcohol consumption. Studies were eligible for review if the study population were defined as TYA cancer survivors and the mean age of the sample was younger than 30 years of age.

Results: Twelve studies were identified, of which nine were randomized controlled trials. Physical activity was the most commonly targeted health behavior. Six of the 12 interventions included within this review were successful in changing health behavior. Due to the heterogeneity of intervention characteristics, the relationship between intervention efficacy or outcome and intervention content, delivery mode, or theoretical framework was not discernible. Nevertheless, trends emerged relating to the delivery and content of health behavior interventions designed specifically for TYA cancer survivors.

Conclusion: More research is required to identify the most effective means of promoting health behavior change among the TYA cancer survivor population. Specifically, future research should focus on providing evidence of the efficiency and feasibility of interventions that use online technologies to facilitate remote intervention delivery and peer support.

Keywords: physical activity, diet, tobacco use, alcohol use, risk behavior, survivorship

\section{(}

$\mathbf{T}$ HE NUMBER OF TEENAGE AND YOUNG ADULT (TYA)aged cancer survivors is rising. At the end of 2010, approximately 16,500 TYAs were living with and beyond cancer within the United Kingdom. ${ }^{1}$ This figure is only set to increase given advances in long-term survival rates in this age group. ${ }^{2}$ However, young people often face long-term consequences as a result of their diagnosis and treatment. Not only do TYA-aged cancer survivors face a heightened risk of cancer recurrence, evidence suggests that around $60 \%$ will suffer from at least one additional chronic health condition within their lifetime. ${ }^{3,4}$ The long-term and latent effects of treatment such as cardiovascular disease, endocrine dysfunction, osteoporosis, stroke, and kidney failure pose a significant burden to survivors throughout their life course.,
However, such comorbidities are often progressive and strongly influenced by a myriad of factors, including lifestyle. ${ }^{7}$ Positive health behaviors such as being physically active, adopting a healthy diet, not smoking, and limiting alcohol intake may prevent the onset and development of late effects and reduce the incidence of cancer recurrence within this population. ${ }^{5,6,8-10}$ Evidence from the St. Jude Lifetime Cohort Study has shown that long-term adult survivors of childhood and adolescent malignancies who did not follow physical activity and diet guidelines set out by the World Cancer Research Fund and American Institute for Cancer were more likely to have metabolic syndrome with a relative risk of 2.2 (95\% CI 1.6-3.0) among males and 2.4 (95\% CI 1.7-3.3) among females. ${ }^{11}$ In addition, a recent cross-sectional

\footnotetext{
${ }^{1}$ Health Behaviour Research Centre, Department of Epidemiology and Public Health, University College London, London, United Kingdom.

${ }^{2}$ Communications and Campaigning, CLIC Sargent, London, United Kingdom.

${ }^{3}$ Department of Haematology, University College London Hospital, London, United Kingdom.
} 
comparison that aimed to explore the relationship between physical activity levels and cardiovascular risk factors among TYA-aged cancer survivors and their siblings reported that survivors $\left(N=319 ; M_{\text {age }}=14.6\right.$ years $)$ who were more physically active had a better cardiovascular risk profile than those who were not active. Combined, these findings suggest that physical activity and healthy lifestyle among young cancer survivors may reduce cardiovascular risk and improve long-term health by the same mechanisms observed in older adult cancer survivors and age-matched healthy population controls. $^{12,13}$

However, although evidence is limited, surveys suggest that a significant proportion of TYA-aged cancer survivors fail to meet diet and physical activity recommendations ${ }^{14-17}$ and engage in multiple risky health behaviors such as smoking and drinking. ${ }^{18,19}$ A cross-sectional survey of 74 young adult cancer survivors $\left(M_{\mathrm{age}}=22.9\right.$ years $)$ found that only $48.7 \%$ of the sample met physical activity guidelines and that more than half retrospectively rated their current fitness as "much worse" or "not quite as good" compared with pre-diagnosis. However, in total, $85 \%$ of participants surveyed indicated a desire for information about exercise. ${ }^{20}$ Likewise, in a recent study of 170 TYA survivors $\left(M_{\text {age }}=\right.$ 17.7 years) in the United States, $75 \%$ were interested in participating in weight-control programs, with $84 \%$ indicating an interest in more information on healthy eating. ${ }^{14}$ Combining this level of interest, the fact that cancer diagnosis and treatment is often viewed as a "teachable moment", 7,21 and that teenage and early adult periods are key developmental stages for behavioral intervention, ${ }^{22}$ efforts to support TYA-aged cancer survivors to adopt healthy lifestyle behaviors are therefore urgently required.

The efficacy of health behavior intervention in long-term childhood cancer survivors and adult cancer survivors has been reviewed previously. ${ }^{23-25}$ However, until very recently, TYA-aged cancer survivors have been largely neglected within health behavior research. As a result, very little is currently understood about the best setting and timing to initiate lifestyle intervention among young people affected by cancer. Identifying common characteristics of successful interventions is an important step in informing the development of health behavior interventions designed specifically for this unique cohort of cancer survivors.

The aim of this systematic review was therefore to synthesize the literature published to date on health behavior interventions targeting physical activity, dietary, smoking, and/or alcohol consumption behaviors among TYA-aged cancer survivors.

\section{Methods}

A search was conducted in May 2015 for studies written in English and published in peer-reviewed journals describing the outcome of an intervention targeting health behavior change in TYA cancer survivors.

\section{Search strategy}

Health behaviors of interest were physical activity, diet, tobacco use (smoking), and alcohol consumption (and all permutations of these behaviors). The following search terms were applied to CINAHL PLUS, Embase, Medline, and PsyhInfo bibliographic databases: cancer patient* or cancer survivor* AND aerobic* or exercis* or fitness or gym* or physical* or sport* or diet* or energy intake or food* or fruit* or nutrition* or veg* not VEGF or drink* or cigarette* or alcohol or smok* or tobacco* AND random* or RCT or trial or intervention AND Teen* or Adolescen* or TYA or AYA or Young Adult. The search strategy was developed in consultation with a research librarian at University College London. Authors of studies selected for review were contacted to enquire as to whether they knew of any additional health behavior interventions targeting TYA cancer survivors conducted as a randomized controlled trial (RCT) or otherwise, which were either published or unpublished.

\section{Selection criteria}

As this systematic review was part of a program of research aiming to develop a health behavior intervention for TYA-aged cancer survivors, it was necessary that the principal summary measure reported within included manuscripts was health behavior change (i.e., magnitude of increase or decrease in the frequency of targeted health behaviors). Participants of interest were those between 13 and 24 years of age defined as a teenager, adolescent, or young adult cancer survivor. However, given inconsistencies in the TYA age bracket between countries, and in order to capture all relevant literature, studies wherein (i) participants were described as TYA cancer survivors and (ii) the mean age of the participant group fell below 30 years were also included for review. Given the lack of behavioral research within this area, intervention studies wherein the mean age of participants fell slightly above or below the lower and upper ends of this criterion bracket were also included for review. In addition, no studies were excluded based upon participants' treatment status or position upon the cancer care continuum. This is in alignment with the National Cancer Institute, World Cancer Research Fund, and American Institute of Cancer Research definition of cancer survivor as anyone who has had a cancer diagnosis from the time point of diagnosis until the end of life: this includes those who are undergoing treatment and those who have recovered from the disease. ${ }^{26-28}$

\section{Data collection}

As shown in Figure 1, 1311 studies were identified within the initial search. The titles and abstracts of all articles were individually screened by the first author to assess eligibility for inclusion. Full-text versions of articles that potentially met the inclusion criteria were subsequently retrieved and screened to assess eligibility for inclusion. After independent screening, included and excluded studies were reviewed by G.P. and A.F., and any discrepancies were resolved by discussion. A total of 12 intervention studies, comprising 13 articles, were included for full review. The data and information regarding sample characteristics, intervention design methodology, and intervention outcomes pertaining to health behavior change were extracted from the original studies using predesigned extraction forms. Data from each study were summarized and aggregated. Meta-analysis was not possible due to the wide variation of outcome measures used within studies.

\section{Study quality}

The methodological quality of the included RCT studies was measured using the Cochrane Collaboration risk of bias 


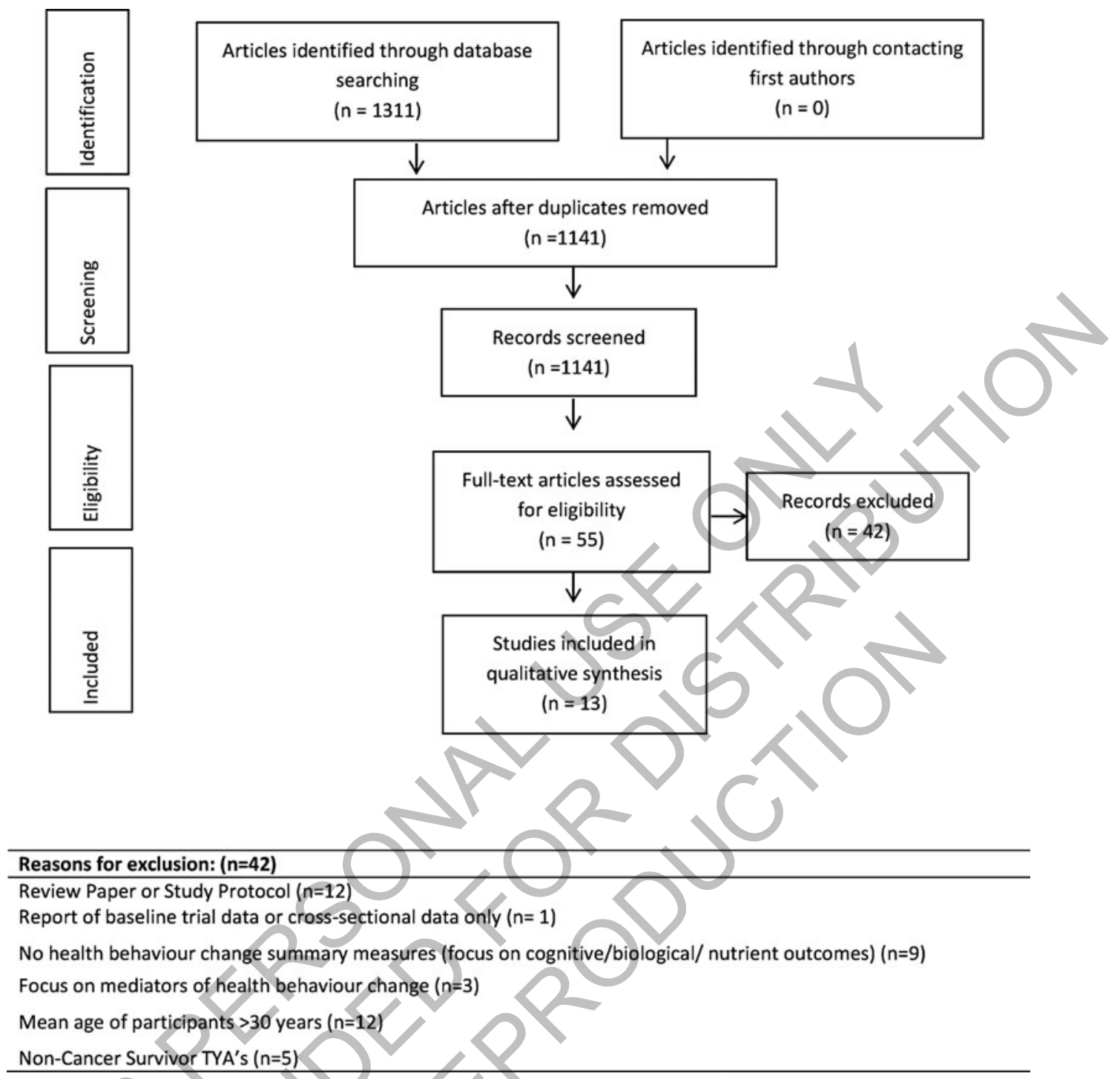

FIG. 1. Flow diagram of literature search and reasons for exclusion.

tool, ${ }^{29}$ which allows evaluation of study quality based on six key criteria: random sequence generation, allocation concealment, blinding, incomplete outcome data, selective reporting, and other sources of bias. Each study was rated low risk, unclear, or high risk for each category of bias. Within this review, a study was considered to have a high risk of bias if two thirds or more of the criteria were scored as high risk.

\section{Results}

Characteristics of included studies are summarized in Table 1. Of the 12 studies included, 75\% $(n=9)$ were twoarmed RCTs, with the remainder being either one-armed feasibility trials $(n=2)^{30,31}$ or repeated-measures longitudinal designs $(n=1))^{32}$ The majority of studies $(n=9 ; 75 \%)$ were conducted in the United States, ${ }^{31-40}$ with the remaining three studies $(25 \%)$ being conducted in Canada, ${ }^{41}$ China, ${ }^{42}$ and Finland. ${ }^{30}$

In total, 1209 participants $(n=509$ male, $n=700$ female) were recruited across all studies. The smallest sample included 10 participants, ${ }^{32}$ and the largest $266 .{ }^{33,37}$ Typically, partici- pants were between 13 and 18 years of age. However, three studies specifically recruited young adult participants older than 18 years of age. ${ }^{31,40,41}$ All studies included both female and male participants, with most studies successfully recruiting more females than males. Ten of the 12 studies included reported details of ethnicity. In these, exact numbers were generally not given, but the majority of participants recruited were reported to be white/Caucasian, with the remainder of participants being African American, Latino, Hispanic, or Asian.

Cancer survivors of hematological malignancies were the most commonly recruited, with two studies ${ }^{30,36}$ specifically recruiting acute lymphoblastic leukemia (ALL) survivors $(n=55)$. Within studies wherein time since diagnosis, time since treatment, or age at diagnosis was reported, it could be inferred that the majority of participants across the included studies were diagnosed with cancer during their childhood before the age of 12 years.

The primary aim of the majority of studies was to establish the feasibility and efficacy of novel interventions promoting health behavior change among TYA-aged cancer survivors. Five intervention studies solely aimed to change physical 


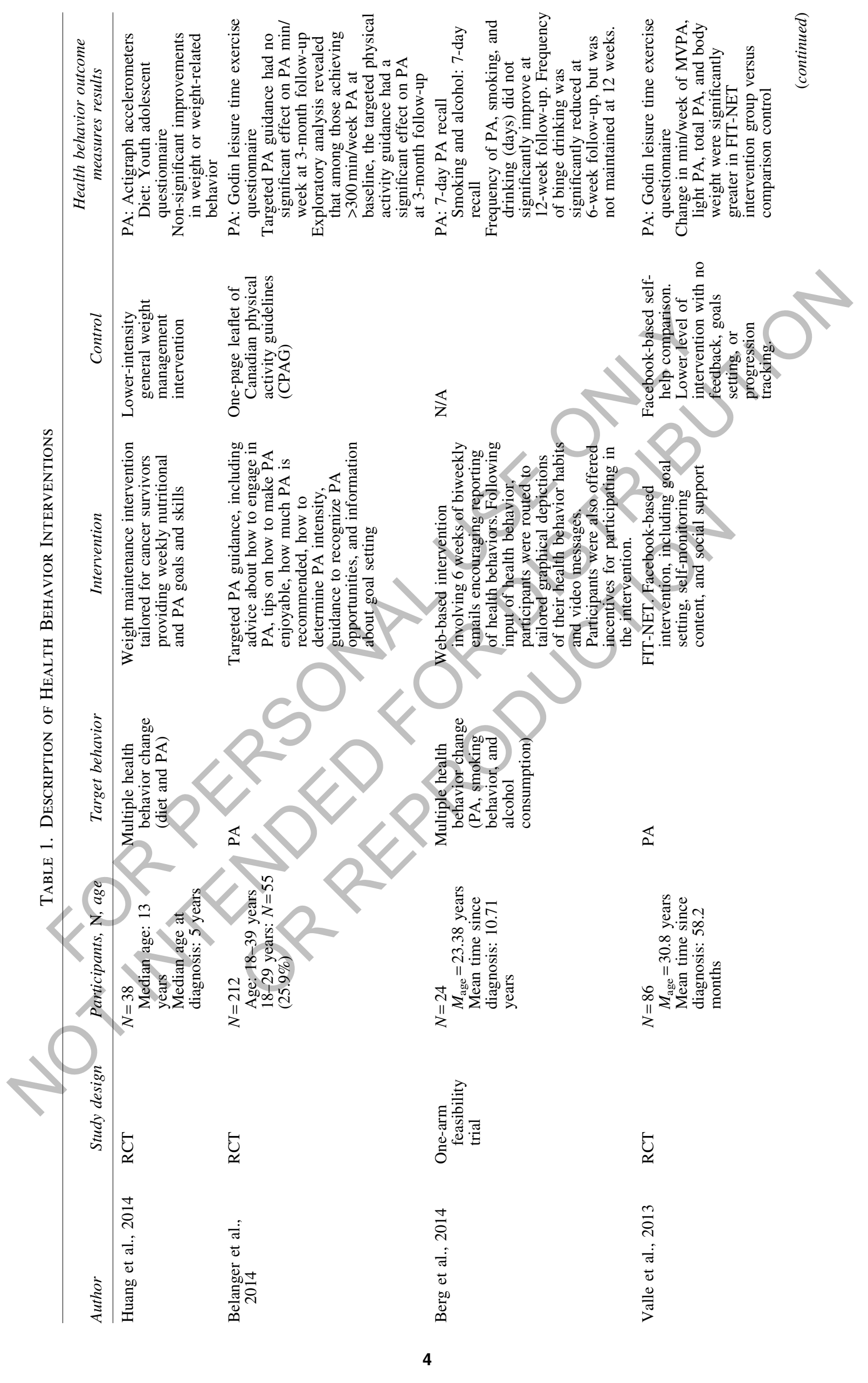




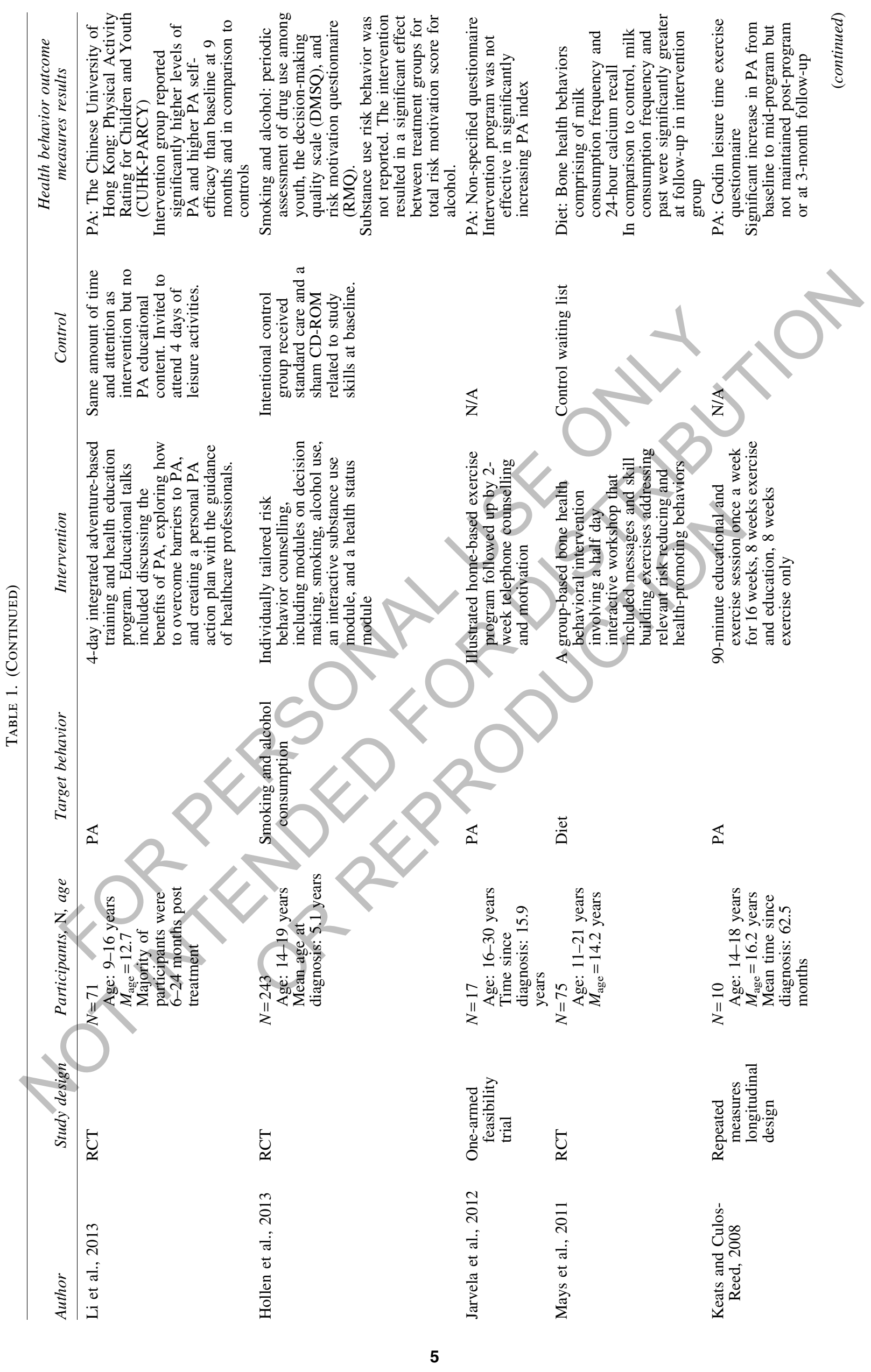




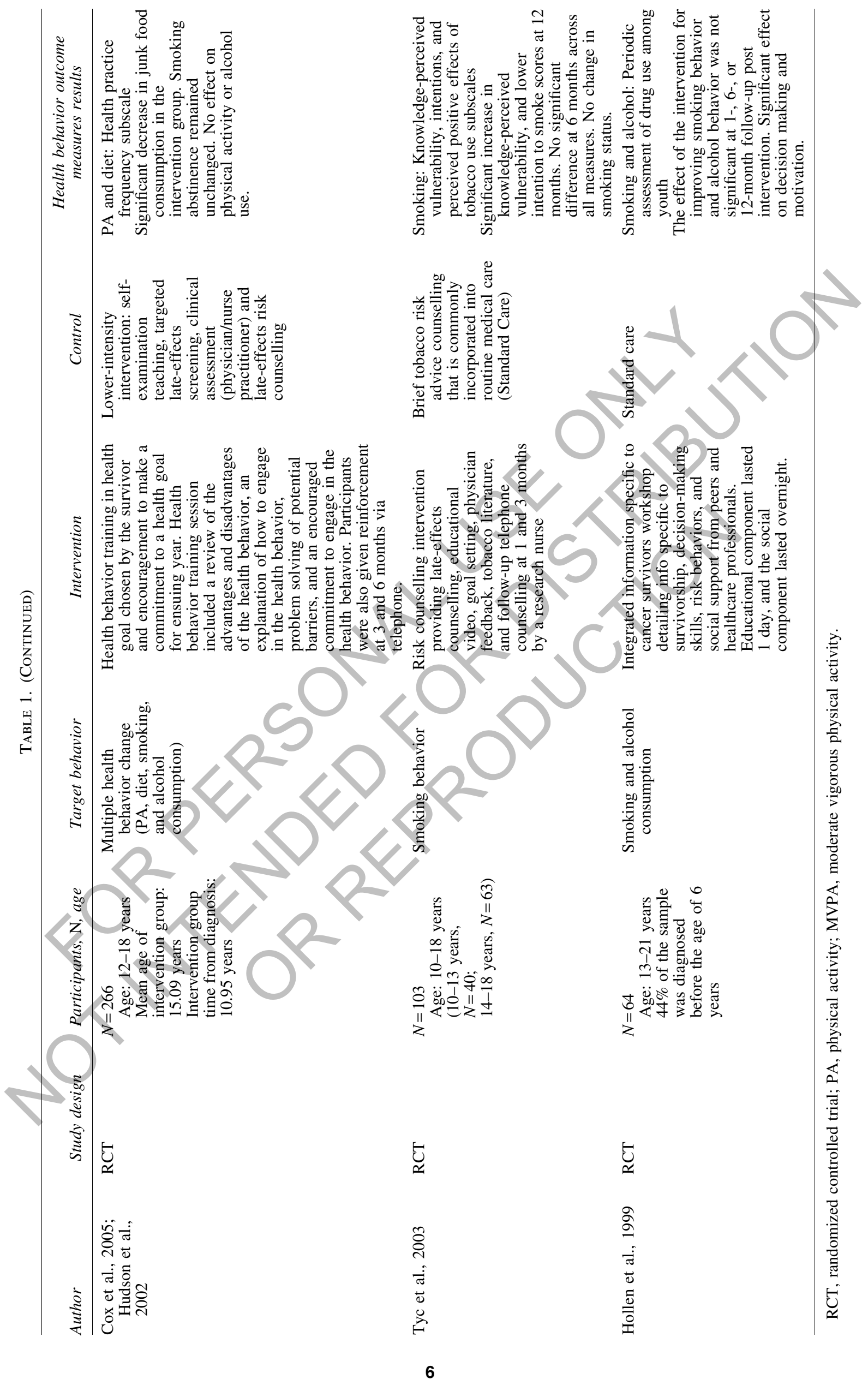




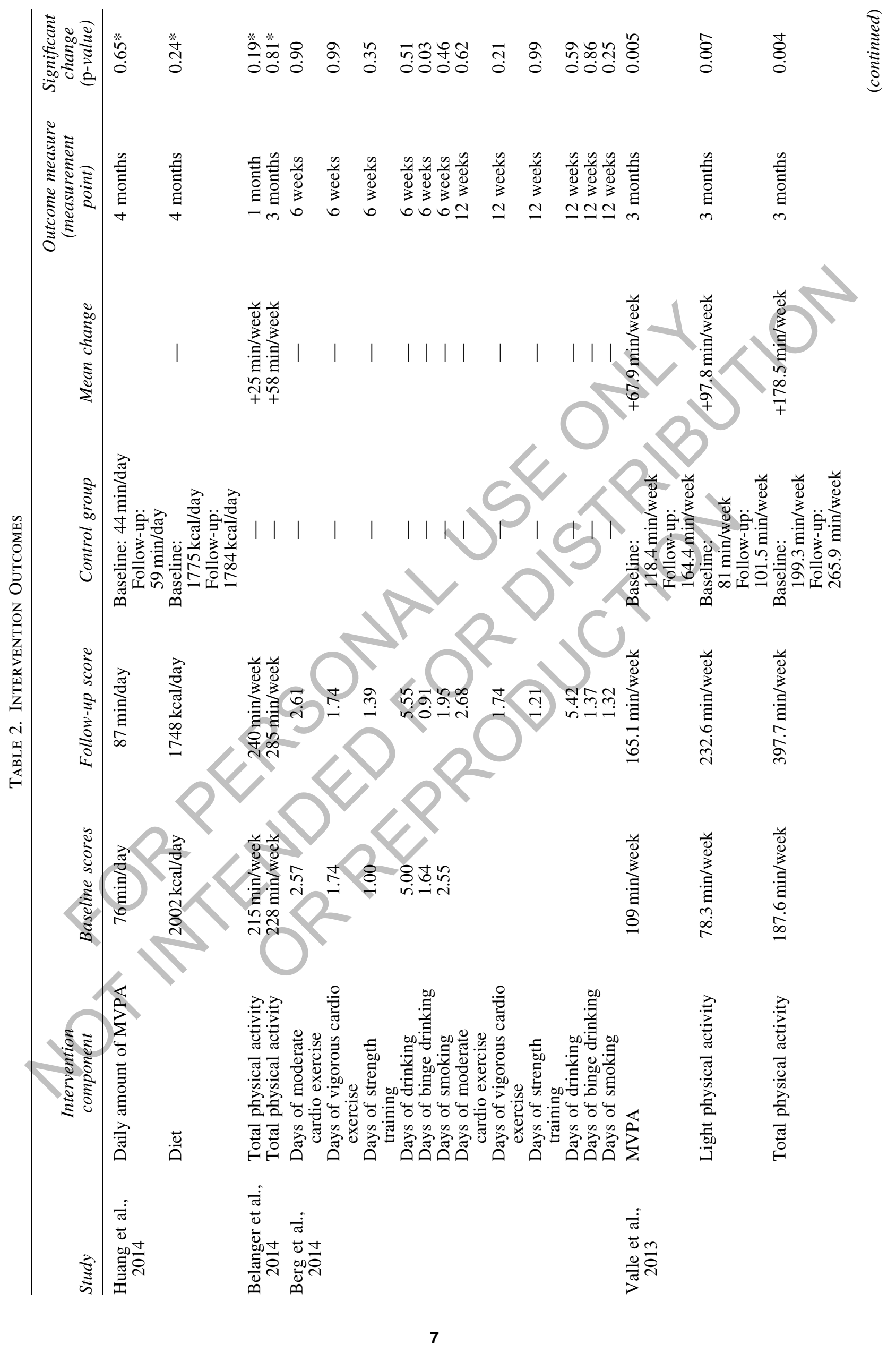




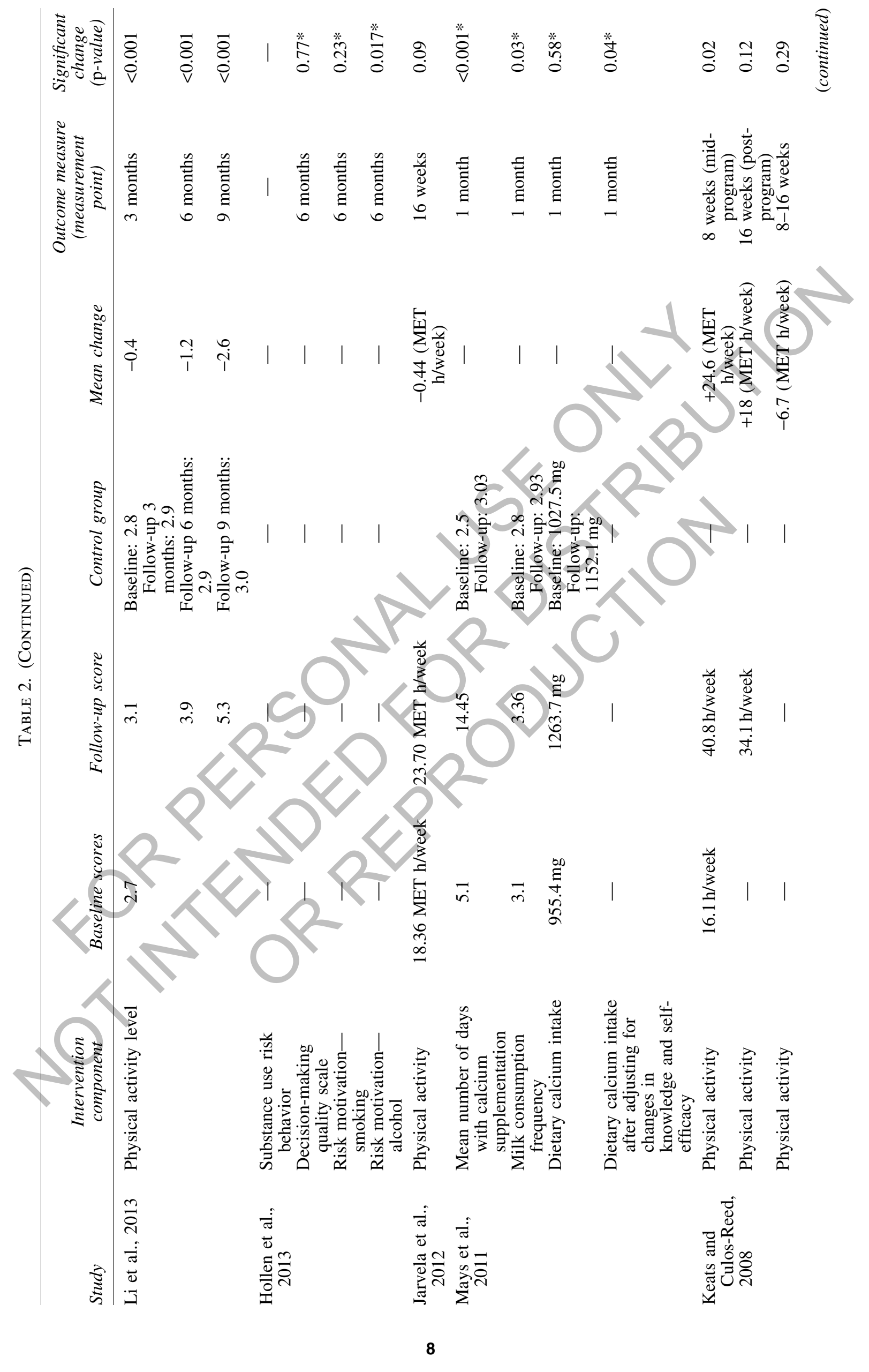




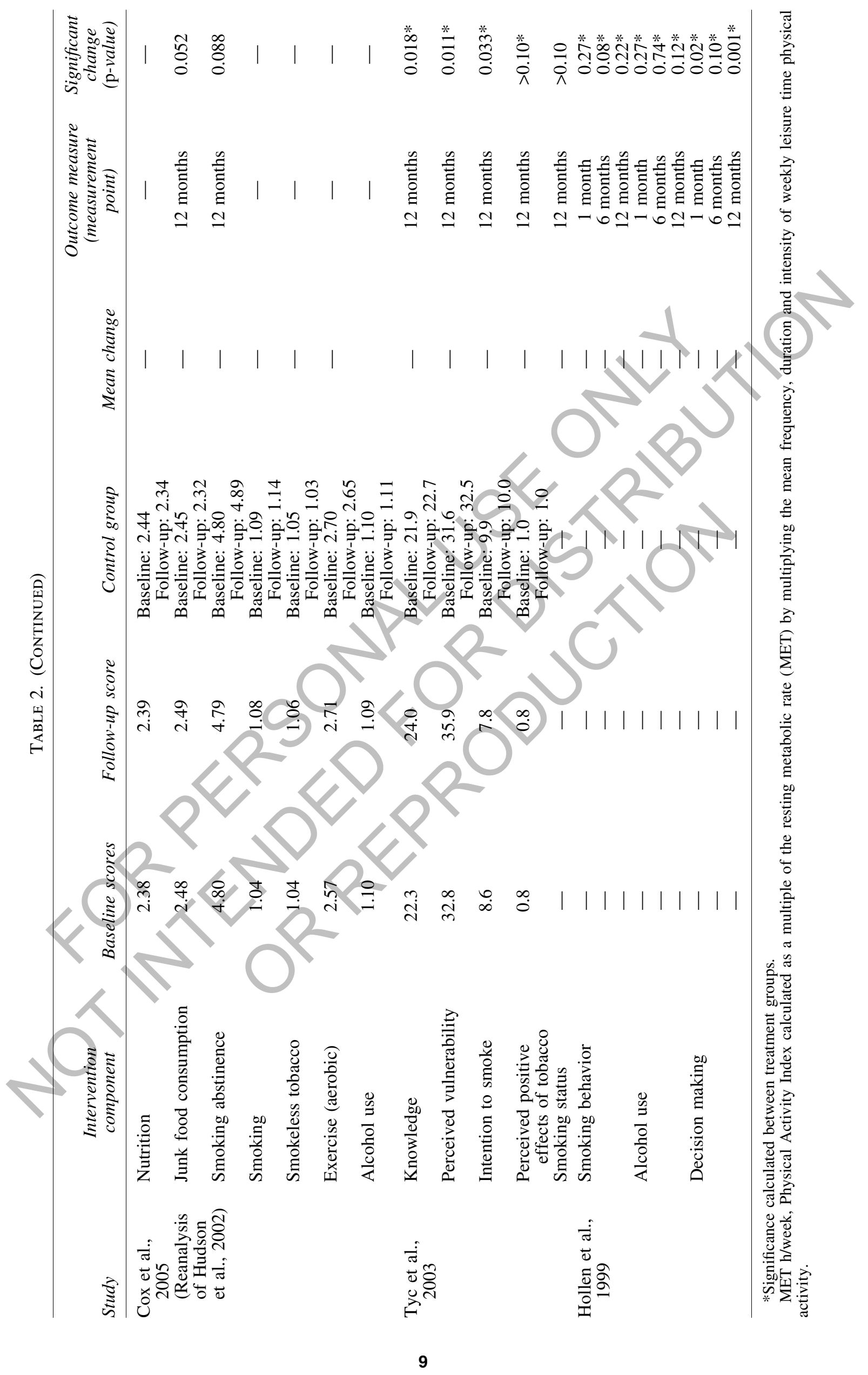


activity, $^{30,32,40-42}$ one study addressed diet and nutrition, ${ }^{38}$ one addressed smoking and tobacco use ${ }^{39}$ and five addressed multiple health behaviors in unison. ${ }^{33-37}$ Of the five studies that addressed multiple health behaviors, one targeted physical activity and diet, ${ }^{36}$ two addressed smoking and alcohol consumption, ${ }^{34,35}$ and two addressed physical activity, diet, smoking and alcohol health behaviors. $31,33,37$

Table 2 displays the outcomes of all included interventions. Of the 12 included studies, all were deemed to be practical and feasible methods of promoting behavior change among young people who had been diagnosed with cancer. However, only six $(50 \%)$ were successful in significantly changing target health behavior. $^{31-33,38,40,42}$ Reported mean change in health behavior from baseline or difference between intervention group and control was typically small. Three intervention studies, although not achieving significant behavior change, did have a significant effect on mediators of health behavior. ${ }^{34,35,39}$

Table 3 outlines component features and delivery methods of included interventions. There was wide variation in the combination of component features used within the interventions contained within this review. As a result, collectively reviewing the content and component features of all studies was difficult, given the heterogeneity of intervention characteristics and outcome measures. Nevertheless, patterns and trends relating to intervention delivery emerged.
Half $(n=6)$ of all included studies promoted health behavior change remotely, meaning the intervention was delivered without face-to-face interaction with the researchers conducting the intervention. Within five $\mathrm{e}^{30,33,34,36,37,39}$ studies, phone calls were used as a means to provide additional counselling or motivation to intervention participants at various time points throughout the intervention. A third $(n=4)$ of the interventions were computer based and delivered online via specifically designed Websites, ${ }^{31}$ CD-ROMs, ${ }^{35}$ existing social networking Websites, ${ }^{40}$ or email reminders. Only one intervention used text messaging to deliver intervention reminders. ${ }^{36}$ Four interventions included day-long workshops that aimed to facilitate health behavior change by facilitating informed decision making, ${ }^{34}$ creation of an action plan, ${ }^{42}$ and skill-building exercises. ${ }^{38,42}$ Printed materials were often used to support information received during workshops or counselling sessions.

Ten of the 12 interventions incorporated an aspect of emotional or social support, in the form of one-to-one contact with a research nurse, $34,35,39$ facilitated group sessions, ${ }^{38,42}$ or the opportunity to interact with other young people who have also had a diagnosis of cancer. ${ }^{40}$ Only one study targeted health behavior change with the support of the family. ${ }^{36}$ Half $(n=6)$ of the interventions ${ }^{31,33,35,36,39,42}$ provided individually tailored information to the participant in the

Table 3. Component Features of All Included Studies

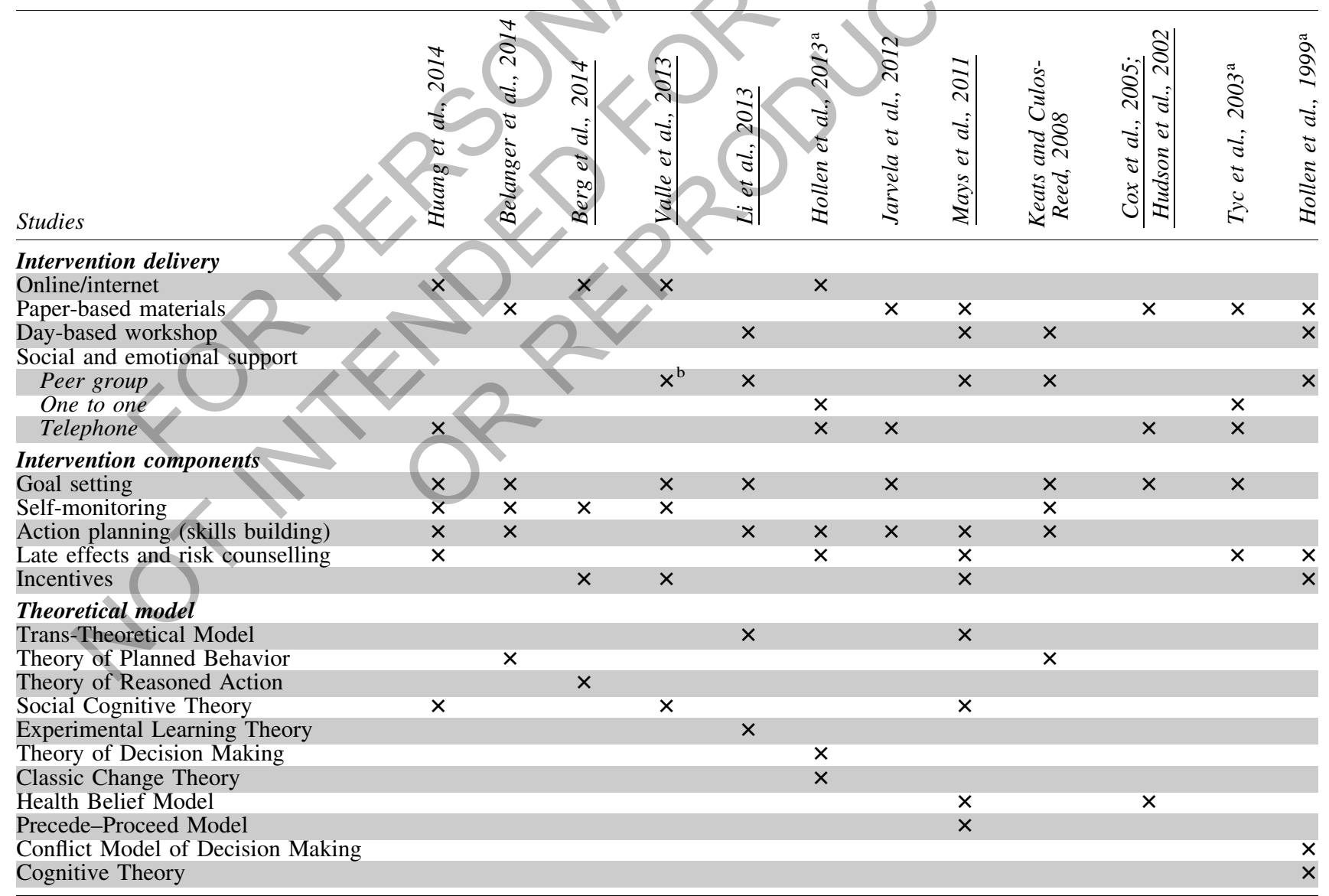

Studies underlined are those that were successful in changing one or more health behavior.

${ }^{2}$ Studies that had a significant effect on mediators of health behavior.

${ }^{\mathrm{b}}$ Peer-group support was delivered using an online social networking Website. No face-to-face communication took place. 
form of personalized behavioral counselling, feedback, or motivation. Online platforms (e.g., private Facebook groups) were utilized as novel methods of incorporating aspects of social support into the intervention. ${ }^{40}$

The content and format of four interventions had been developed and designed by the researchers working in consultation with young people. ${ }^{31,36,38,41}$ The intervention content of one study was guided by a specially commissioned advisory group. ${ }^{42}$ A range of theoretical models were used as a basis for behavior change in many of the interventions (Table 3). One third $(n=4)$ of the intervention studies $^{34,36,38,39}$ included within this review provided education and personalized risk counselling regarding the latent effects of cancer diagnosis and treatment to participants.

The duration of interventions ranged from a single halfday group session to 12 months where intervention materials were delivered at scheduled time points. The number of contacts (including delivery of intervention, baseline, and follow-up measures) made by the research team to the participant also varied across studies, but was not associated with the duration of the intervention study. Interventions that were delivered remotely using modern communication technologies such as the internet or telephone typically had more information delivery contact points than interventions delivered by other methods.
Due to heterogeneity between intervention characteristics, no clear relationship could be drawn between intervention efficacy or outcome and intervention content, delivery, theoretical design, or duration. However, of the interventions that significantly changed behavior, five out of six included social support in the form of peer group interaction, one-to-one counselling, or telephone support from a nurse, health coach, or member of the research team conducting the intervention. Interventions that were successful in achieving significant behavior change were also heavily incentivized. Within studies where participant evaluation was reported, most interventions were generally received positively by young people.

A breakdown of the methodological quality classifications for each included RCT is displayed in Table 4. Only one $\mathrm{RCT}^{42}$ was deemed to be at low risk of bias, with the remaining being deemed to be at moderate risk of bias.

\section{Discussion}

The findings of this systematic review highlight the novel and diverse range of intervention design and delivery methods used to promote health behavior change among TYAaged cancer survivors. Six of the 12 interventions included within this review were successful in changing health behavior. However, the relationship between intervention

Table 4. Scores for Risk of Bias Assessment (Cochrane Collaboration Tool) for All InCluded RCT Health Behayior Interventions $(N=9)$

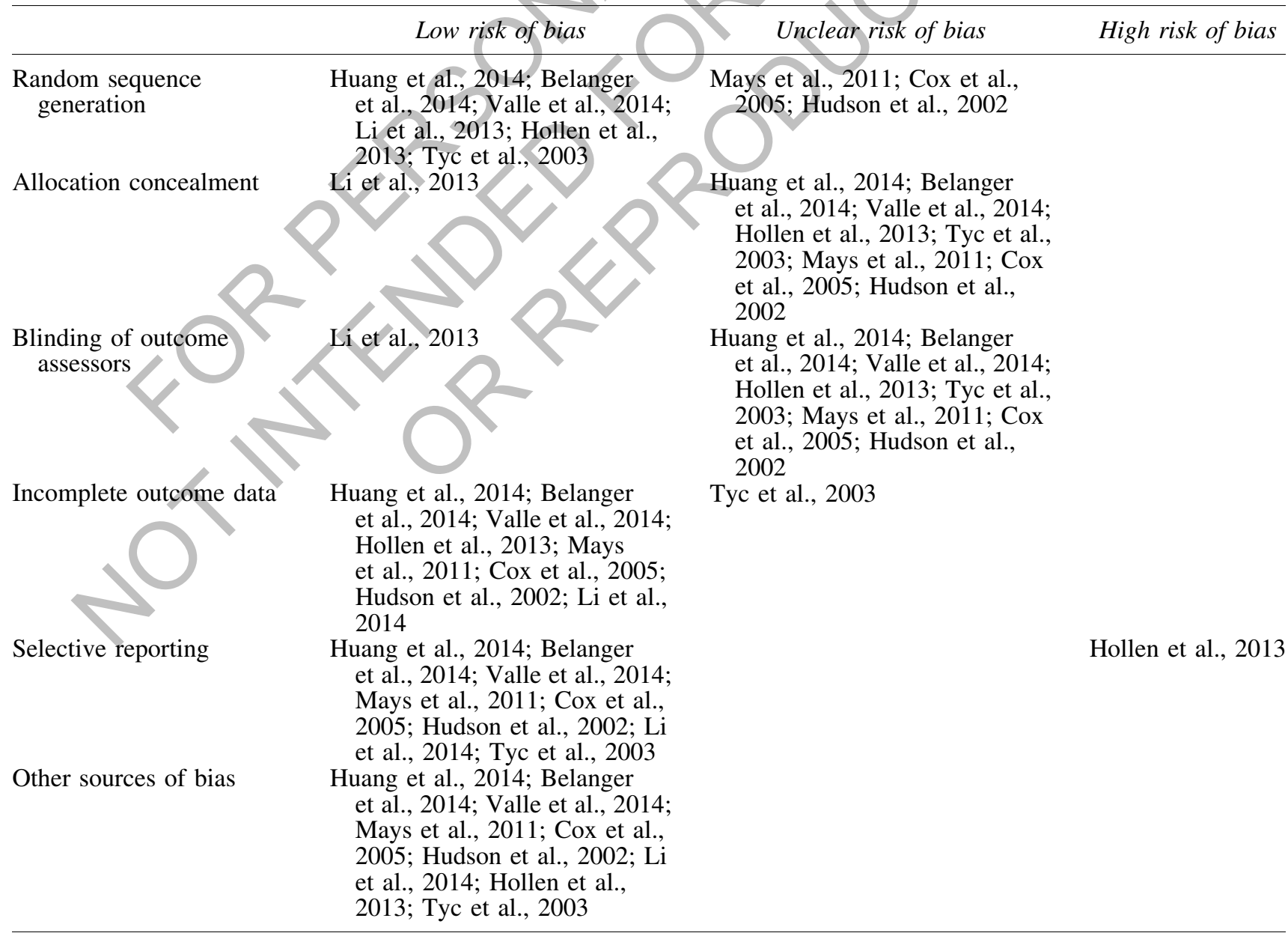


efficacy or outcome and intervention content, delivery mode, or theoretical design was undiscernible due to the heterogeneity of intervention characteristics. Although the number of eligible studies identified was limited due to the lack of research within this area, trends emerged relating to the delivery and content of health behavior interventions designed specifically for TYA-aged cancer survivors.

Half of interventions were delivered remotely. The majority of remotely delivered interventions were either computer based or involved a mail out of paper-based intervention materials. This is in keeping with previous studies investigating the intervention preferences of TYA-aged cancer survivors. ${ }^{14,15,43}$ Remote intervention delivery within this population may be especially beneficial, given that TYA cancer survivors often have time consuming obligations (e.g., studying and working) and physical limitations (e.g., chronic fatigue) that may limit their engagement and interest in health promotion activities. $^{44-46}$

Two intervention studies included within this review used online features of pre-existing social network websites and specifically designed websites to deliver the health behavior intervention to young people. The reach of the internet in communicating health information to young people is vast and online platforms of communication are increasingly becoming an effective means of facilitating social support in efforts to promote healthy lifestyle behaviors among this hard to reach population. ${ }^{47,48}$ Websites and mobile applications (apps) have emerged as promising and innovative approaches to remote intervention delivery. Previous studies of the internet features most desired by TYA cancer survivors found that young people want websites that contain both age appropriate cancer specific information and opportunities to communicate with other cancer survivors. ${ }^{49}$ Specifically designed lifestyle websites and mobile phone apps which create an easily accessible and enriched information environment (e.g. additional videos, links, or testimonials), facilitate behavior tracking, tailor feedback, and provide an aspect of peer support are worth further investigation within this context. In addition utilization of online recruitment strategies may overcome the recruitment and adherence challenges typically faced by researchers within this area. $^{50,51}$

Nevertheless, although interventions delivered remotely are emerging as promising strategies to lifestyle promotion among this age group, social, emotional, and instrumental support from healthcare professionals, friends, and family has been identified as a major feature of health behavior change among adolescents and young adults. ${ }^{52}$ Ten out of 12 studies contained at least one aspect of social support. Although the variation in social support formats between interventions meant social support could not be attributed to intervention efficacy or outcome, support in the form of peer interaction or counselling delivered either in person or via the telephone was a key feature of many health behavior interventions included within this review.

It is important to note lifestyle choice is a multidimensional construct with many interpersonal, intrapersonal, and environmental factors influencing an individual's health behavior. ${ }^{53}$ Previous studies support this notion and have found social influences to be independent predictors of health behavior and lifestyle choice among TYA-aged cancer survivors. In one study of adolescents cancer survivors aged 12-16 years, family and peer support for physical activity was found to be a significant predictor of time spent in moderate to vigorous physical activity. Conversely, only family physical activity levels and family support for physical activity were found to be predictors of physical activity among younger cancer survivors aged $8-12$ years. ${ }^{54}$ Having friends with poor diets has also previously been cited as a barrier to healthy eating by young people who have experienced cancer. ${ }^{44}$ As in healthy adolescent and young adult population groups, as a young person who has had a diagnosis of cancer transitions from adolescence to adulthood, family influence upon behavior decreases, and young people often seek direct and indirect support from close friends or peers. Efforts to deliver health information and support to TYA-aged cancer survivors should take into account this transitional phenomenon and potentially target social groups as mediators of behavior change.

The incorporation of personalized risk counselling regarding the latent effects of cancer diagnosis and treatment into the health education component of the intervention was a common feature in several studies. Explicitly emphasizing health risk and long-term consequences of cancer diagnosis and treatment has been identified as alternative means of promoting health behavior change among young adult survivors, ${ }^{55}$ as greater understanding of risk may motivate young people to adopt healthier lifestyles. ${ }^{56}$ Such interventions that support the development of autonomous motivation and decision making may be more effective in promoting health behavior change among TYA cancer survivors than strategies and theories that take a more directive approach to changing knowledge and beliefs. ${ }^{57}$ Future intervention studies should aim to quantify the impact of risk counselling upon participants motivation and self-efficacy to health behavior change.

A large proportion of studies included within this review addressed physical activity as a health behavior. However, more insight is required in relation to smoking, alcohol consumption, and, specifically, dietary health behavior change among TYA cancer survivors. It is important that future intervention studies also discriminate between health protective behaviors (such as physical activity and diet) and harmful health behaviors (such as smoking), and establish if different approaches must be taken to address these individual behaviors.

Although there is evidence that theory-based health promotion interventions may help improve intervention outcome ${ }^{58,59}$ this review could not demonstrate a link between the theoretical basis and the outcome of any included studies. Nevertheless, interventions that were designed in consultation with young cancer survivors showed promising results. The inclusion of TYA cancer survivors within research design is an important step in advocating patient centered care that supports and respects young people's autonomy and independence. $^{60}$

This review highlights the diverse range of health behavior interventions that are practical and viable ways of promoting a healthy lifestyle among young people who have had a cancer diagnosis. No studies included within this review reported any adverse outcomes. Many intervention studies included within this review reported promising results, suggesting that if the intervention was replicated with sufficient power, significance could be achieved. Although some 
studies $^{35,40}$ reported that compliance to the intervention protocol was low, this was typically anticipated, and strategies such as incentives were used to maximize participant attrition. In one particular intervention study, despite a third of participants not complying fully with the booster features of the intervention, $90 \%$ rated all evaluation criterion positively. ${ }^{35}$ Overall, participant enthusiasm and engagement with the intervention studies was high. This is reflective of the interest young people have previously reported in health behavior information. ${ }^{14,20}$

Cancer diagnosis and treatment are often viewed as a "teachable moment" for behavior change because of the unique impact diagnosis and treatment have upon an individual's motivation to learn about, adopt, and maintain positive health behaviors. ${ }^{59,61}$ However, in practice, there is little evidence in adult populations that cancer survivors spontaneously adopt healthier lifestyles post diagnosis without intervention. ${ }^{62}$ Very little is currently understood about whether the "teachable moment" is also a point in TYA survivorship and if this point is related to stage of treatment, age, or individual. Further investigation into the information and support preferences of young people who have had a cancer diagnosis is required to establish at what time point within the cancer journey health behavior intervention should be delivered. Equally, further investigations into the efficacy and feasibility of promoting lifestyle change among TYAaged cancer survivors is required to clarify the practicality of delivery modes and intervention contents that are easy to disseminate, cost-effective, and offer age appropriate advice to young people who have had a diagnosis of cancer. In turn, this will provide evidence of the most effective means of promoting health behavior change among TYA-aged cancer survivors.

Several limitations in intervention study design and reporting of results must be addressed. Brief descriptions of intervention design within studies limited the stratification of potential effective components of the intervention and limited the interpretation of methodological quality, with few studies proving clear information of the randomization procedure or blinding at outcome assessment. When considering the results of the interventions as a collective, it is important to note the small sample size and variable adherence rates that limit the power to detect significant effects in analyses within several studies. The generalizability of the reported outcome is also limited, as hematological malignancies, although common, only account for a small amount of the variety of cancer diagnoses in TYA cancer survivors. In addition, few studies followed had a long-term follow-up of health behavior changes, making it unrealistic to draw conclusions about the long-term efficacy of these interventions to produce durable health behavior changes. In addition, most behavior change outcomes were measured subjectively, suggesting that results should be interpreted with caution, considering evidence that young people are proxy to high levels of response bias, specifically during the self-report of smoking and alcohol behaviors. A small number of potentially informative studies may also have been excluded from analysis, despite including young people between 13 and 24 years of age, as the mean age of the sample may have been older than 30 years. Specific research into the health behaviors and lifestyle choices of TYA cancer survivors is limited, and hence many of the citations used within this review may in fact refer to TYA-aged childhood cancer survivors. Care has been taken where necessary to emphasize this.

\section{Conclusion}

Adopting a healthy lifestyle is central to improving the health and quality of life of young people who have had a cancer diagnosis. Supporting and encouraging TYA-aged cancer survivors to lead a healthy lifestyle may help reduce the impact of treatment late effects upon both their current and their long-term health. While several interventions designed specifically for TYA cancer survivors have shown promising results, further work is required to evaluate how best to promote health behavior change to young people who have had a cancer diagnosis. Equally, evaluation of the impact of behavior change on TYA cancer survivors' longterm health as they continue into adulthood is also required. Future interventions should explore the use of e-health technologies to support behavior change and provide further insight into the feasibility and acceptability of such interventions. Development of such interventions should occur in partnership with both TYA cancer consumer groups and TYA cancer professionals in order to ensure that interventions are developed and designed to have the greatest impact.

\section{Acknowledgments}

This work was supported in part by an IMPACT Award cofunded by University College London and CLIC Sargent awarded to Gemma Pugh.

\section{Author Disclosure Statement}

No competing financial interests exist.

\section{References}

1. National Cancer Intelligence Network (NCIN). MacmillanNCIN work plan. Segmenting the cancer survivor population by cancer type, 20 year prevalence at the end of 2010 , UK. Accessed October 2015 from: www.ncin.org.uk/ view?rid $=2493$

2. O'Hara C, Moran A, Whelan JS, et al. Trends in survival for teenagers and young adults with cancer in the UK 1992-2006. Eur J Cancer. 2015;51(14):2039-48.

3. Mulrooney DA, Yeazel MW, Kawashima T, et al. Cardiac outcomes in a cohort of adult survivors of childhood and adolescent cancer: retrospective analysis of the Childhood Cancer Survivor Study cohort. BMJ. 2009;339;b4606.

4. Ozono S, Ishida Y, Honda M, et al. General health status and late effects among adolescent and young adult survivors of childhood cancer in Japan. Jpn J Clin Oncol. 2014; 44(10):932-40.

5. Robison LL, Hudson MM. Survivors of childhood and adolescent cancer: life-long risks and responsibilities. Nat Rev Cancer. 2014;14(1):61-70.

6. Slater ME, Ross JA, Kelly AS, et al. Physical activity and cardiovascular risk factors in childhood cancer survivors. Paediatr Blood Cancer. 2015;62(2):305-10.

7. Demark-Wahnefried W, Aziz NM, Rowland JH, Pinto BM. Riding the crest of the teachable moment: promoting longterm health after the diagnosis of cancer. J Clin Oncol. 2005;23(24):5814-30. 
8. Doyle C, Kushi LH, Byers T, et al. Nutrition and physical activity during and after cancer treatment: an American Cancer Society guide for informed choices. CA Cancer J Clin. 2006;56(6):323-53.

9. Gritz ER, Demark-Wahnefried W. Health behaviors influence cancer survival. J Clin Oncol. 2009;27(12):1930-2.

10. Tonorezos ES, Robien K, Eshelman-Kent D, et al. Contribution of diet and physical activity to metabolic parameters among survivors of childhood leukaemia. Cancer Causes Control. 2013;(24):313-21.

11. Smith WA, Li C, Nottage KA, et al. Lifestyle and metabolic syndrome in adult survivors of childhood cancer: a report from the St. Jude Lifetime Cohort Study. Cancer. 2014;120(17):2742-50.

12. McTiernan A. Mechanisms linking physical activity with cancer. Nat Rev Cancer. 2008;8(3):205-11.

13. Slater ME, Steinberger J, Ross JA, et al. Physical activity, fitness, and cardiometabolic risk factors in adult survivors of childhood cancer with a history of hematopoietic cell transplantation. Biol Blood Marrow Transplant. 2015;21(7): 1278-83.

14. Badr H, Chandra J, Paxton RJ, et al. Health-related quality of life, lifestyle behaviors, and intervention preferences of survivors of childhood cancer. J Cancer Surviv. 2013;7(4):523-34.

15. Demark-Wahnefried W, Werner C, Clipp EC, et al. Survivors of childhood cancer and their guardians. Cancer. 2005; 103(10):2171-80.

16. Hansen J HS, Klesges L, Tyc V, et al. Eating behaviour and BMI in adolescent survivors of brain tumor and acute lymphoblastic leukemia. J Pediatr Oncol Nurs. 2014;31(1):9.

17. Berdan CA, Tangney CC, Scala C, Stolley M. Childhood cancer survivors and adherence to the American Cancer Society Guidelines on Nutrition and Physical Activity. $\mathbf{J}$ Cancer Surviv. 2014;8(4):671-9.

18. Frobisher C, Lancashire ER, Reulen RC, et al. Extent of alcohol consumption among adult survivors of childhood cancer: the British Childhood Cancer Survivor Study. Cancer Epidemiol Biomarkers Prev. 2010;19(5):1174-84.

19. Tercyak KP, Donze JR, Prahlad S, et al. Multiple behavioral risk factors among adolescent survivors of childhood cancer in the Survivor Health and Resilience Education (SHARE) program. Pediatr Blood Cancer, 2006;47(6):825-30.

20. Murnane A, Gough K, Thompson K, et al. Adolescents and young adult cancer survivors: exercise habits, quality of life and physical activity preferences. Supp Care Cancer. 2015; 23(2):501-10.

21. Ganz PA. A teachable moment for oncologists: cancer survivors, 10 million strong and growing! J Clin Oncol. 2005; 23(24):5458-60.

22. Sawyer SM, Afifi RA, Bearinger LH, et al. Adolescence: a foundation for future health. Lancet. 2012;379(9826):1630-40.

23. Barnes MJ, Demark-Wahnefried W. Importance of balanced diet and physical activity during and after cancer treatment in adolescent patients. Clin Oncol Adolesc Young Adults. 2014:(4)13-20.

24. Brier MJ, Schwartz LA, Kazak AE. Psychosocial, healthpromotion, and neurocognitive interventions for survivors of childhood cancer: a systematic review. Health Psych. 2015; 34(2):130-48.

25. Stolley MR, Restrepo J, Sharp LK. Diet and physical activity in childhood cancer survivors: a review of the literature. Ann Behav Med. 2010;39(3):232-49.

26. National Cancer Institute (NCI). Definition of survivorNCI dictionary cancer terms-National Cancer Institute.
Accessed November 2015 from: www.cancer.gov/dictionary? cdrid $=450125$

27. World Cancer Research Fund and American Institute Cancer Research. Food, nutrition, physical activity and the prevention of cancer: a global perspective. Washington, DC: AICR; 2007.

28. American Cancer Society. Cancer glossary-survivor. Accessed November 2015 from: www.cancer.org/cancer/ cancerglossary/index

29. Higgins JPT, Altman DG, Gøtzsche PC, et al. The Cochrane Collaboration's tool for assessing risk of bias in randomised trials. BMJ. 2011;343:d5928.

30. Jarvela LS, Kemppainen J, Niinikoski H, et al. Effects of a home-based exercise program on metabolic risk factors and fitness in long-term survivors of childhood acute lymphoblastic leukemia. Pediatr Blood Cancer. 2012;59(1):155-60.

31. Berg CJ, Stratton E, Giblin J, et al. Pilot results of an online intervention targeting health promoting behaviors among young adult cancer survivors. Psychooncology. 2014;23(10): 1196-9.

32. Keats MR, Culos-Reed SN. A community-based physical activity program for adolescents with cancer (project TREK): program feasibility and preliminary findings. J Pediatr Hematol Oncol. 2008;30(4):272-80.

33. Cox CL, McLaughlin RA, Rai SN, et al. Adolescent survivors: a secondary analysis of a clinical trial targeting behavior change. Pediatr Blood Cancer. 2005;45(2):144-54.

34. Hollen PJ, Hobbie WL, Finley SM. Testing the effects of a decision-making and risk-reduction program for cancersurviving adolescents. Oncol Nurs Forum. 1999;26(9): 1475-86.

35. Hollen PJ, Tyc VL, Donnangelo SF, et al. A substance use decision aid for medically at-risk adolescents: results of a randomized controlled trial for cancer-surviving adolescents. Cancer Nurs. 2013;36(5):355-67.

36. Huang JS, Dillon L, Terrones L, et al. Fit4Life: a weight loss intervention for children who have survived childhood leukemia. Pediatr Blood Cancer. 2014;61(5):894-900.

37. Hudson MM, Tyc VL, Srivastava DK, et al. Multicomponent behavioral intervention to promote health protective behaviors in childhood cancer survivors: The Protect Study. Med Pediatr Oncol. 2002;39(1):2-11.

38. Mays D, Black JD, Mosher RB, et al. Efficacy of the Survivor Health and Resilience Education (SHARE) program to improve bone health behaviors among adolescent survivors of childhood cancer. Ann Behav Med. 2011;42(1):91-8.

39. Tyc VL, Rai SN, Lensing $\mathrm{S}$, et al. Intervention to reduce intentions to use tobacco among pediatric cancer survivors. J Clin Oncol. 2003;21(7):1366-72.

40. Valle CG, Tate DF, Mayer DK, et al. A randomized trial of a Facebook-based physical activity intervention for young adult cancer survivors. J Cancer Surviv. 2013;7(3):355-68.

41. Belanger LJ, Mummery WK, Clark AM, Courneya KS. Effects of targeted print materials on physical activity and quality of life in young adult cancer survivors during and after treatment: an exploratory randomized controlled trial. J Adolesc Young Adult Oncol. 2014;3(2):83-91.

42. Li HCW, Chung OKJ, Ho KY, et al. Effectiveness of an integrated adventure-based training and health education program in promoting regular physical activity among childhood cancer survivors. Psychooncology. 2013;22(11): 2601-10.

43. Rabin C, Simpson N, Morrow K, Pinto B. Intervention format and delivery preferences among young adult cancer survivors. Intern J Behav Med. 2013;20(2):304-10. 
44. Arroyave WD, Clipp EC, Miller PE, et al. Childhood cancer survivors' perceived barriers to improving exercise and dietary behaviors. Oncol Nurs Forum. 2008;35(1):121-30.

45. Gotte M, Kesting S, Winter C, et al. Comparison of selfreported physical activity in children and adolescents before and during cancer treatment. Pediatr Blood Cancer. 2014;61(6):1023-8.

46. Wright M, Bryans A, Gray K, et al. Physical activity in adolescents following treatment for cancer: influencing factors. Leuk Res Treatment. 2013;(2013):592395.

47. Elliot DL, Lindemulder SJ, Goldberg L, et al. Health promotion for adolescent childhood leukemia survivors: building on prevention science and ehealth. Pediatr Blood Cancer. 2013;60(6):905-10.

48. Gray NJ, Klein JD, Noyce PR, et al. Health informationseeking behaviour in adolescence: the place of the internet. Soc Sci Med. 2005;60(7):1467-78.

49. Schiffman JD, Csongradi E, Suzuki LK. Internet use among adolescent and young adults (AYA) with cancer. Pediatr Blood Cancer. 2008;51(3):410-15.

50. Flanagan S, Greenfield S, Coad J, Neilson S. An exploration of the data collection methods utilised with children, teenagers and young people (CTYPs). BMC Res Notes. 2015;8(1):61.

51. Rabin C, Horowitz S, Marcus B. Recruiting young adult cancer survivors for behavioral research. J Clin Psychol Med Settings. 2013;20(1):33-6.

52. Biggs B, Lebow J, Smith C, et al. Adolescents' preferences for social support for healthful eating and physical activity. J Dev Behav Pediatr. 2014;35(8):494-509.

53. Fitzgerald N. Barriers to a healthy lifestyle: from individuals to public policy - an ecological perspective. J Extension. 2009;47(1): 1 .

54. Gilliam MB, Madan-Swain A, Whelan K, et al. Social, demographic, and medical influences on physical activity in child and adolescent cancer survivors. J Pediatr Psychol. 2012;37(2):198-208.

55. Tyc VL, Hudson MM, Hinds $\mathrm{P}$. Health promotion interventions for adolescent cancer survivors. Cogn Behav Pract. 1999;6(2):128-36.
56. Ford JS, Puleo E, Sprunck-Harrild K, et al. Perceptions of risk among childhood and young adult cancer survivors who smoke. Supp Care Cancer. 2014;22(8):2207-17.

57. D'Agostino NM, Penney A, Zebrack B. Providing developmentally appropriate psychosocial care to adolescent and young adult cancer survivors. Cancer. 2011;117(S10): 2329-34.

58. Pinto BM, Floyd A. Theories underlying health promotion interventions among cancer survivors. Semin Oncol Nurs. 2008;24(3):153-63.

59. Keats MR, Culos-Reed SN, Courneya KS, McBride M. Understanding physical activity in adolescent cancer survivors: an application of the theory of planned behavior. Psychooncology. 2007;16(5):448-57.

60. Zebrack B. Patient-centered research to inform patientcentered care for adolescents and young adults (AYAs) with cancer. Cancer. 2014;120(15):2227-9.

61. Demark-Wahnefried W, Peterson B, McBride C, Lipkus I, Clipp E. Current health behaviors and readiness to pursue life-style changes among men and women diagnosed with early stage prostate and breast carcinomas. Cancer. 2000; 88(3):674-84.

62. Williams K, Steptoe A, Wardle J. Is a cancer diagnosis a trigger for health behaviour change? Findings from a prospective, population-based study. Br J Cancer. 2013;108(11): 2407-12.

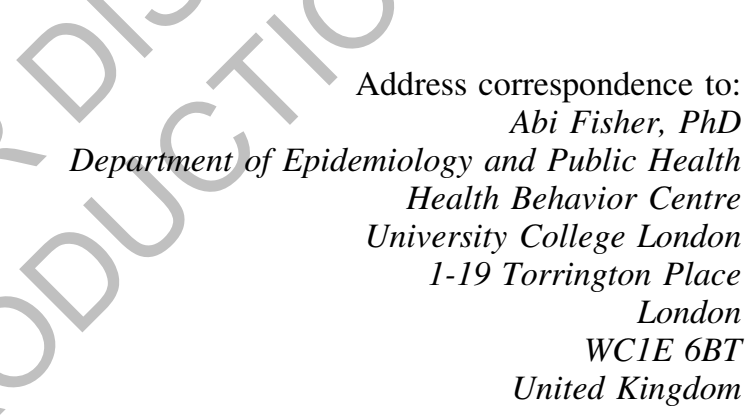

Email: abigail.fisher@ucl.ac.uk 